\title{
The new Italian map at 1:50.000 scale: M793 Map Series
}

\author{
Giuseppe Giustolisi ${ }^{\text {a, }}$ \\ ${ }^{a}$ Italian Geographic Military Institute (IGMI), giuseppe.giustolisi@esercito.difesa.it
}

Keywords: mapping, topography, cartography, portrayal, simbology, national mapping, military map.

\begin{abstract}
:
The Italian Geographic Military Institute (IGMI) began its activity as the Topographic Office of the Italian Army General Staff after the Italian unification, in 1861. It became the national cartographic authority with law no. 68/1960, and since then its maps have been considered the Italian official state cartography.
\end{abstract}

The IGMI cartographic production is focused on land maps at medium scale $(1: 25.000,1: 50.000)$ and small scale (1:250.000, 1:1.000.000) for civilian and military purposes.

The main cartographic series at scale 1:50.000 is M792, the series designation follows the NATO Standardization Agreement (STANAG) 7136 edition 1 (February 2004): the first element "M" is the letter designating the western European Regional Area, the second element "7" is the number that indicates the map scale $(1: 50.000)$, the third element "9" stands for the Italian sub-region and the fourth element " 2 " is the number that indicates the edition $\left(2^{\wedge}\right.$ Edition).

The M792 was completed for the whole national territory in 2018 and since then IGMI has started to develop a new map edition, the M793, based on vector data collected in the "Data Base di Sintesi Nazionale" (DBSN, [National Synthesis Data Base]) and compliant with the Multinational Geospatial Co-Production Program (MGCP) Topographic Map (MTM) specifications. The MTM is a paper map characterized by a high level of planimetric detail and quantitative representation of the relief through contour lines. The various features shown on the map are represented by standard symbols. These symbols are explained at the bottom of the map along with other information about the map and its contents. Topographic map's contents include symbolization of transports and cultural features, hydrography, hypsography, vegetation, boundaries, geographic toponyms, together with a Military Grid Reference System (MGRS).

The M793 map's workflow has required the transition from the Italian national Data Product Specification (DPS), that is the DBSN data schema, to the requirements of the MGCP Technical Reference Documentation (TRD) ver. 4.4 in order to meet symbol and portrayal rules of the MTM. After the schema conversion, there is a generalization process of geometries from the nominal scale at 1:25.000 to the 1:50.000 scale; for example, buildings geometry is changed from polygon to point and buildings within a built-up area are grouped.

The geodetic reference system is ETRS89 (European Terrestrial Reference System 1989) in the realization ETRF2000 (European Terrestrial Reference Frame 2000) - epoch 2008. The M793 map series is made of elements, called "sheets" as for the previous series. Each sheet has a dimension of 20' Longitude x 15' Latitude. The series consists of 568 sheets. Sheets are numbered in compliance with MTM specifications (3.14.2.3 Numbering of map sheets) following the division into sheets at scale 1:100.000 and each sheet is systematically identified by a four-digit number. The four-digit number is composed of two significant pairs of digits in which the first one identifies the column and the second one the row. The westernmost column of the sheets is assigned the value 10 (first two digits) as well as the southernmost row (second two digits).

To comply with MTM specifications (3.14.2.1-Establishment of standard sheet lines) the 1:50.000 scale sheet lines are based on a 1:100.000 scale format, which has been designed to incorporate the relevant paper map series at a worldwide level, in particular one sheet at scale 1:100.000 consists of four sheets at scale 1:50.000.

This presentation will cover off each M793 map's workflow phase and will also introduce how IGMI will represent the Italian territory in the future, thanks to this new map series. 